\title{
Surfactant adsorption at liquid/liquid interfaces Comparison of experimental results with self-consistent field lattice calculations and molecular dynamics simulations
}

\author{
N.M. Van Os ${ }^{a}$,*, L.A.M. Rupert ${ }^{\mathrm{b}}$, B. Smit ${ }^{\mathrm{a}}$, P.A.J. Hilbers ${ }^{\mathrm{a}}$, K. Esselink ${ }^{\mathrm{a}}$, M.R. Böhmer ${ }^{\mathrm{c}}$, \\ L.K. Koopal ${ }^{\mathrm{c}}$ \\ ${ }^{a}$ Koninklijke/Shell-Laboratorium, Amsterdam (Shell Research BV), Badhuisweg 3, 1031 CM Amsterdam, \\ The Netherlands \\ ${ }^{\mathrm{b}}$ Thornton Research Centre, P.O. Box 1, Chester, UK \\ ${ }^{\mathrm{c}}$ Wageningen Agricultural University, Department of Physical and Colloid Chemistry, Dreijenplein 6, 6703 HB \\ Wageningen, The Netherlands
}

(Received 12 March 1993; accepted 14 June 1993)

\begin{abstract}
A comparison of experimental data with self-consistent field lattice calculations and molecular dynamics simulations has shown that the latter two approaches are able to predict in a qualitative sense the relation between the structure of a surfactant and its interfacial tension at an oil/water interface. Micelles can also be observed in the simulations and in the self-consistent field calculations. Advantages and disadvantages of the simulations and the self-consistent field calculations are discussed and it is concluded that current theoretical models provide reasonable descriptions of complex colloidal systems.
\end{abstract}

Key words: Adsorption; Interface; Molecular dynamics; Self-consistent field; Surfactant

\section{Introduction}

Detergency and improved water flooding to enhance oil recovery can be defined, in a simplified sense, as the removal of oily soil, or oil, from a solid surface (a fibre or reservoir rock) by the action of a surfactant or a mixture of surfactants. This process can, perhaps somewhat naively, be described in terms of a contact angle problem which, in combination with Young's law [1], leads to the requirements that the interfacial tension (IFT) between the oil and the aqueous phase, and the IFT between the solid phase and the aqueous phase should be small.

*Corresponding author.
Gibbs' adsorption equation [2] then tells us that this lowering of the IFT can be brought about by surfactant adsorption at both interfaces.

Virtually all surfactants that have been considered for detergency and improved water flooding show excess adsorption at the solid/aqueous solution interface. Of course, this is of great economic concern in enhanced water flooding, where this interface can be very large. As a consequence, loss of surfactant through adsorption may make such processes uneconomical.

At the oil/aqueous solution $(o / w)$ interface, excess surfactant adsorption will lead to the IFT being lowered. The IFT of an oil/water/surfactant system is closely related to phase behaviour [3]. 
Systematic studies relating IFTs to the structure of surfactant molecules are needed to provide insight into what happens at the molecular level. It is here that in recent years theoretical developments have come to the fore which can give such insight, and which may also aid the synthetic chemist in tailoring new compounds.

Broadly speaking, such theoretical developments can be classified into two categories. Firstly, statistical mechanical descriptions have received widespread attention [4-18]. A well-known example is the self-consistent field lattice theory for adsorption and/or association (SCFA), originally developed for the study of polymer adsorption [4-6], but recently extended to describe systems with small chain molecules [7-10]. Membrane and micelle formation has been studied [8-10] and also the adsorption of amphiphilic molecules at liquid/ liquid [11] and solid/liquid interfaces [12-15]. In the same category of mean field theories we have those developed by Gruen [16] and by Szleifer and co-workers $[17,18]$. Secondly, molecular dynamics simulations [19-25] have been used to study oil/water/surfactant systems; either all the atomic details are taken into account [19-21], or the model is simplified to the extent that only essential features are retained [22-25].

In the present paper we are interested in gaining insight into the relation between the structure of a surfactant and its capability of reducing the IFT of a bare oil/water interface. We are interested, in particular, in the effects of extending and branching the hydrophobic chain of the surfactant. In order to study these structural changes in a systematic way, we have conducted an experimental investigation of a series of surfactants with increasing chain length, and of a series with increasing degree of chain branching. In order to gain insight at the molecular level, we have used self-consistent mean field calculations and molecular dynamics (MD) simulations of related model systems.

\section{Experimental studies}

The effect of hydrophobe chain length and hydrophobe chain branching on the IFT of a decane/water interface was studied with two series of surfactants, namely sodium $p$-(3-alkyl)benzenesulphonates with the alkyl group being nonyl, decyl, or dodecyl, and with sodium $p$-( $x$-decyl)benzenesulphonates with $x=2,3$ or 5 . The structures of these compounds are shown in Fig. 1; micelle formation of these and related compounds have been described in detail elsewhere [26-29]. These compounds contain at least $96 \%$ of the parasubstituted isomer, the remainder being some ortho- and/or meta-substituted isomers, or water. The IFT was determined at $25^{\circ} \mathrm{C}$ as a function of surfactant concentration by means of a semi-automatic Lauda tensiometer (du Nouy ring method). The effect of increasing the chain length of these surfactants on IFT has been published elsewhere [23]. The results are shown in Fig. 2 as plots of the interfacial tension against $\log$ (concentration). Longer tails are more effective at low concentration, i.e. less surfactant is needed to achieve a
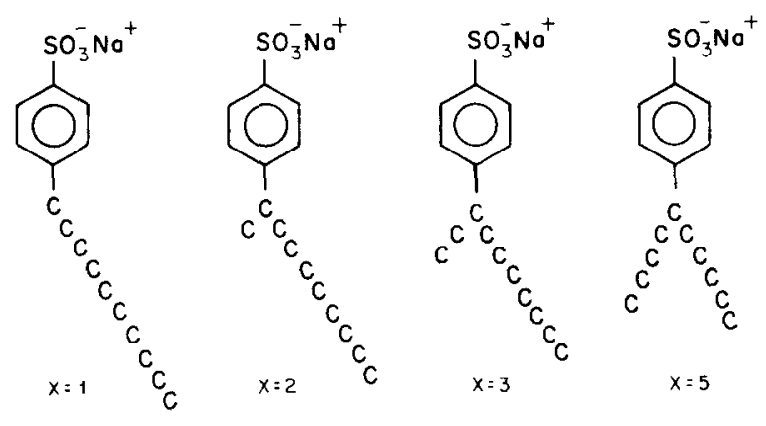

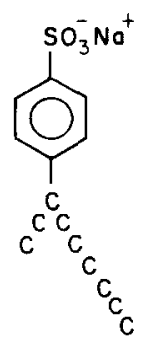

NONYL

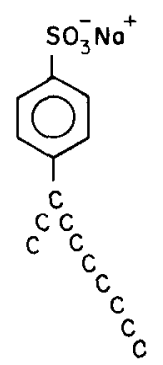

DECYL

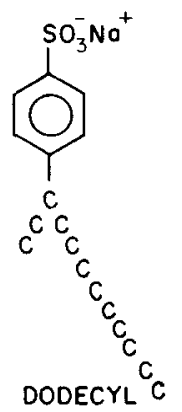

Fig. 1. Structure of sodium $p$-( $x$-alkyl)benzenesulphonates used in the experimental determination of the IFT. Top, $(x$-decyl)series; bottom, (3-alkyl)series. 


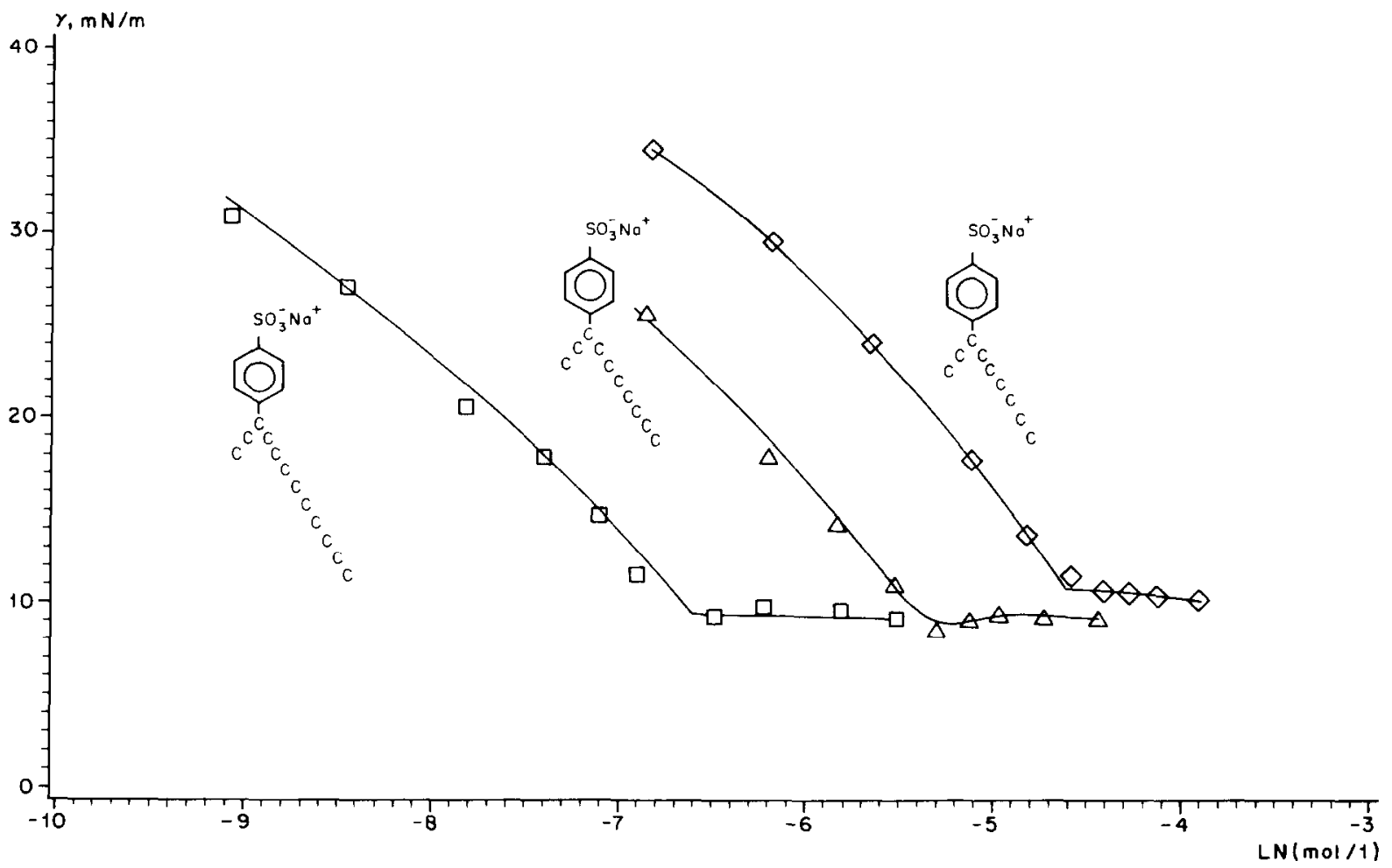

Fig. 2. The interfacial tension vs. the logarithm of surfactant concentration for sodium $p$-(x-alkyl)benzenesulphonates at $25^{\circ} \mathrm{C}$.

given IFT value. The break in the curves as shown in Fig. 2 is due to the formation of micelles in the water phase. The critical micelle concentrations (CMC), which can be deduced from the plots in Fig. 2, are shown in Table 1 together with $\mathrm{CMC}$

Table 1

Critical micelle concentrations of sodium $p$-( $x$-alkyl)benzenesulphonates at $25^{\circ} \mathrm{C}$

\begin{tabular}{lccc}
\hline$x$-alkyl & $\begin{array}{l}\mathrm{CMC}^{\mathrm{a}} \\
\left(\mathrm{mmol} \mathrm{l}^{-1}\right)\end{array}$ & $\begin{array}{l}\mathrm{CMC}^{\mathrm{b}} \\
\left(\mathrm{mmol} \mathrm{1}^{-1}\right)\end{array}$ & $\begin{array}{l}\mathrm{CMC}^{\mathrm{c}} \\
\left(\mathrm{mmol} \mathrm{kg}^{-1}\right)\end{array}$ \\
\hline 3-nonyl & 10.4 & 10.3 & 12.1 \\
3-decyl & 4.6 & 6.0 & 5.0 \\
3-dodecyl & 1.4 & 2.2 & 1.3 \\
2-decyl & 4.1 & 4.6 & 4.8 \\
5-decyl & 7.5 & 8.0 & - \\
\hline
\end{tabular}

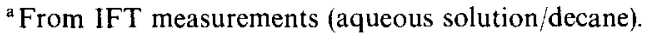

${ }^{b}$ From electrical conductivity measurements [26,27].

${ }^{\circ}$ From enthalpy of dilution measurements at $30^{\circ} \mathrm{C}$ [28].

${ }^{\mathrm{d}}$ Impossible to determine. values derived from electrical conductivity measurements $[26,27]$ and from enthalpy of micellisation experiments [28]. There is reasonable agreement between CMC values derived from the IFT measurements and those obtained from enthalpy data; CMC values derived from conductivity measurements deviate somewhat and are in most cases higher than the other values. It is known that different experimental methods yield different CMC values, since the concentration at which the micclles first become detectable depends on the experimental sensitivity; a thorough discussion of this subject has been given elsewhere [30]. The descending parts of the curves in Fig. 2 close to the break are nearly parallel; according to Gibbs' adsorption equation this means that the amounts adsorbed (in $\mathrm{mol} \mathrm{m}^{-2}$ ) should be the same. For the $\mathrm{C}_{9}, \mathrm{C}_{10}$ and $\mathrm{C}_{12}$ homologues of Fig. 2 this quantity is $(2.1 \pm 0.1) \times 10^{-6} \mathrm{~mol} \mathrm{~m}{ }^{-2}$. For concentrations exceeding the $\mathrm{CMC}$, the IFT tends to 
increase somewhat with decreasing chain length, its value being $9.4 \mathrm{mN} \mathrm{m}^{-1}$ for the $C_{10}$ and $C_{12}$ homologues, and 10.6 for the $\mathrm{C}_{9}$ compound.

The effect of increasing chain branching is shown in Fig. 3, which is a plot of the interfacial tension against $\log$ (concentration). CMC values of branched compounds are given in Table 1. From the data we conclude that there is not much difference between the 2-decyl and the 3-decyl compounds, but that the 5-decyl compound deviates significantly from the other two in the sense that it leads to higher IFT values in the monomeric region of the plots. From the slope of the plots in Fig. 3 we estimate the amount adsorbed at the CMC to be $(2.2 \pm 0.1) \times 10^{-6} \mathrm{~mol} \mathrm{~m}^{-2}$ for the three isomers. For concentrations exceeding the $\mathrm{CMC}$, the IFT decreases slightly with increasing degree of branching, i.e. from $9.8 \mathrm{mN} \mathrm{m}^{-1}$ (2-decyl compound) to $8.6 \mathrm{mN} \mathrm{m}^{-1}$ (5-decyl compound).

\section{Self-consistent field calculations}

\subsection{The self-consistent field lattice theory for adsorption and/or association ( $S C F$ A)}

For a detailed description of the SCFA theory we refer to previous papers [6-15]. Basically, it is an extension of Flory's polymer solution theory [31], which allows for compositional inhomogeneities in one direction, for instance perpendicular to an interface. The SCFA theory uses a lattice on which the molecules are placed. Chain molecules consist of $r$ segments and occupy $r$ lattice sites. Monomers are assumed to have the size of just one lattice site. The lattice is completely filled with molecules. In the lattice one can distinguish layers; in a flat lattice these layers are parallel to the interface. For adsorption at the liquid/liquid interface we use such a flat lattice. For the calcula-

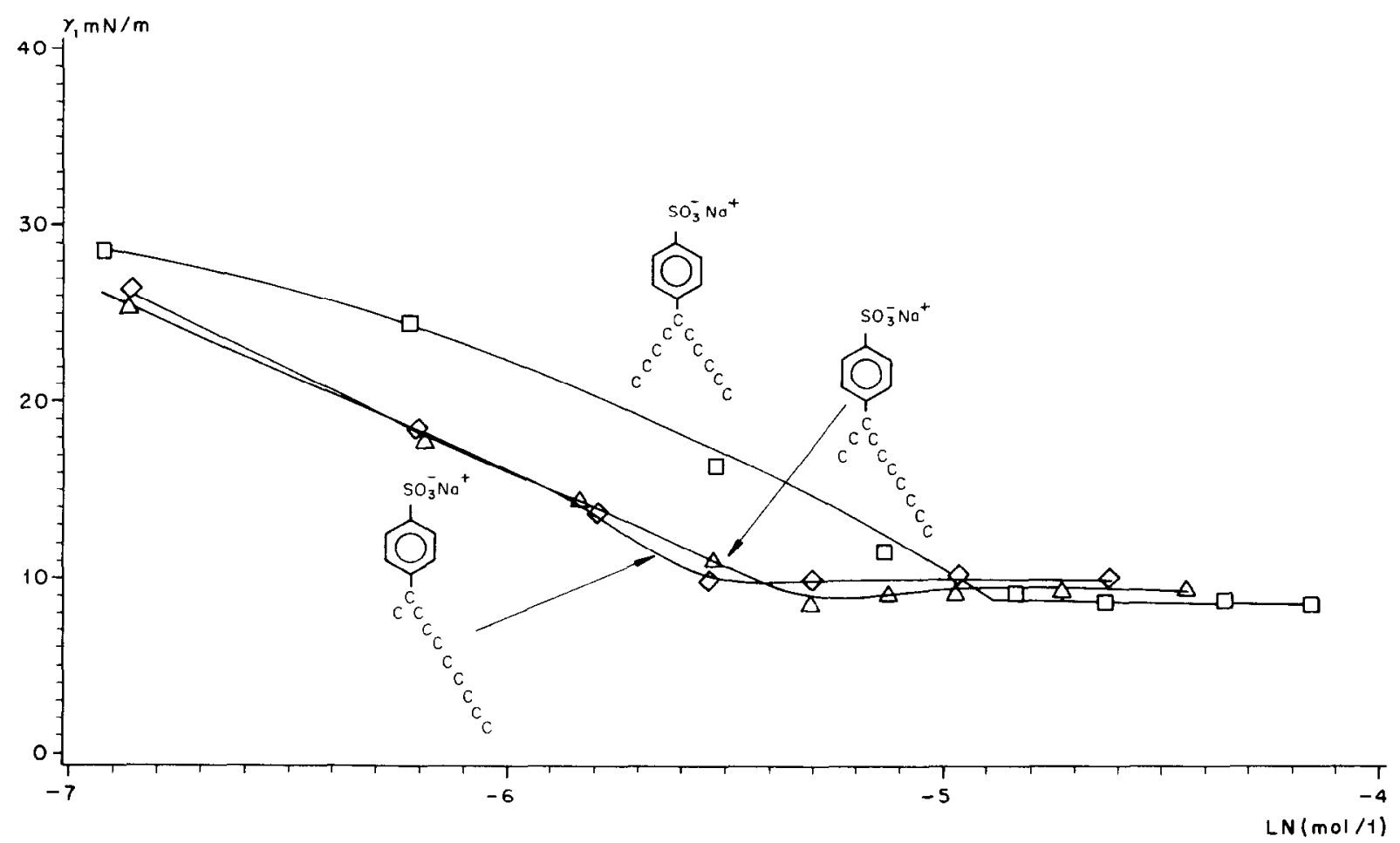

Fig. 3. The interfacial tension vs. the logarithm of surfactant concentration for branched sodium $p$-( $x$-decyl)benzenesulphonates at $25^{\circ} \mathrm{C}$ 
tion of micelles a lattice of spherical symmetry is used [8-10]. Each lattice layer is assumed to be homogeneous.

The conformational statistics of the chain molecules are evaluated by using a rotational isomeric state scheme, as described elsewhere [7,9]. This method permits a distinction to be made between trans and gauche bonds, with backfolding of five subsequent segments being forbidden. Nearest neighbour contact interactions are quantified through Flory-Huggins interaction parameters. The main results from a calculation with the SCFA theory are the volume fraction profiles of the components. These volume fraction profiles give the spatial distribution of molecules perpendicular to the interface. From the volume fraction profiles the interfacial tension can be calculated [11].

\subsection{Choice of parameters}

A simple set of parameters is sufficient to calculate the main features in surfactant adsorption at the liquid/liquid interface. The water is modelled as monomers of type $\mathrm{W}$, the oil as $\mathrm{A}_{4}$ chain molecules, containing four segments of type $A$. The Flory-Huggins interaction parameter $\chi(\mathrm{AW})$ is set at 4.3 , the same value as used before [13]. This high value indicates a strong repulsion and ensures phase separation of $\mathrm{W}$ and $\mathrm{A}_{4}$. The surfactants are modelled as $A_{n} B_{2}$ molecules. $B_{2}$ is the hydrophilic head group and the $\chi(\mathrm{BW})$ value is set at -4.3 . $\mathrm{A}$ consequence of this choice is that the head groups prefer to be surrounded by water rather than by other surfactant head groups. We shall give some results for $\chi(\mathrm{BW})=0$ as well, because this compares better with the molecular dynamics simulations. The interaction between A and B is unfavourable; for the sake of simplicity we chose the same value as for $\chi(\mathrm{AW})$ namely, $\chi(\mathrm{AB})=4.3$. A repulsive interaction between $\mathrm{A}$ and $\mathrm{B}$ promotes micelle formation. With these interaction parameters the effect of aliphatic surfactant chain length on the CMC of non-ionic surfactants can be reproduced assuming that three $\mathrm{CH}_{2}$ units are grouped in one segment [13]. This segment choice differs from the one used in Ref. 10, where one $\mathrm{CH}_{2}$ group was defined as a segment. The present choice is more realistic with respect to the flexibility of the surfactant chain and corresponds better with the choices made in the molecular dynamics simulations. The number of segments is indicated by $r$.

\subsection{Lattice artefacts}

A serious drawback of the lattice theory is that lattice artefacts can occur. Especially for high $\chi$ values the interface between oil and water becomes rather sharp and extends over just a few lattice layers, i.e. the oil/water transition is modelled by only a few discrete steps. Consequently, the results are affected by the localisation of the lattice layers in the interfacial region. For calculations at liquid/liquid interfaces, however, these artefacts can be eliminated. Far away from the interface, where the system is homogeneous, the volume fractions must be the same as those obtained with the Flory equations. Using this as an additional boundary condition, the "artefact-free" surface tension can be calculated. To this end the amount of $\mathrm{A}_{4}$ in the system was varied until the Flory conditions were met.

For the calculation of the IFT at the CMC, which is essential for surfactant behaviour, there is no extra condition available to overcome the effect of lattice artefacts. Therefore, we can only give a rough estimate of the CMC. Since we have used much larger segments and hence larger $\chi$ values than in Ref. 10, the CMCs calculated in the present paper will be less accurate than the earlier values [10].

\subsection{Results}

\subsubsection{Interfacial tension as a function of concentration}

The interfacial tension is expressed in units of $k T$ per area of a lattice site, taking the bulk solution as the reference state. The surfactant concentration is expressed as $\bar{\phi} / r$, with $\bar{\phi}$ being the total volume fraction of surfactant; $\bar{\phi} / r$ is proportional to the 
molar concentration of surfactant. Below the CMC, $\bar{\phi}$ is the volume fraction of free surfactant; above the $\mathrm{CMC}, \bar{\phi}$ is the volume fraction due to the free surfactant and surfactant present in the form of micelles. The results for a homologous series of $\mathrm{A}_{n} \mathrm{~B}_{2}$ surfactants are given in Fig. 4 .

In agreement with the experimental results, the interfacial tension starts to decrease at a lower $\bar{\phi} / r$ value for longer chains and the descending parts of the curves are parallel for the chain lengths studied, indicating that the amount adsorbed in mol $\mathrm{m}^{-2}$ is the same. The curves are truncated at the $\mathrm{CMC}$, indicated with an asterisk. As the uncertainty in the calculated CMC is large (see the bars in Fig. 4), we have refrained from calculations above the CMC. The results do indicate, however, that for concentrations above the CMC, the IFT tends to increase with decreasing chain length of the surfactant, as was also observed experimentally.

Figure 5 gives results for chain molecules that, apart from the size effect, do not have a repulsion between the head groups or a favourable hydration, i.c. $\chi(\mathrm{WB})=0$. In this casc cach head group scgment is water-like. Micelle formation was not observed for these molecules. Aggregates can be formed, but they grow into very large structures instead of small micelles. The formation of these aggregates only begins when the interfacial tension has become negative, which is physically unrealistic for this type of surfactant. Therefore, we may conclude that this choice of parameters does not give the surfactant behaviour observed in the expcriments.

Figure 6 shows the effect of chain branching on interfacial tension. Again, the results agree with the experiment. As branching increases, the slope of the $\gamma-\ln (\bar{\phi} / r)$ plot becomes smaller and the $\bar{\phi} / r$ value at which micelles form becomes higher. The reason for this behaviour is that the more branched molecule packs less efficiently in a dense structure. From the slope of the $\gamma-\ln (\bar{\phi} / r)$ curves it can be deduced that the amount adsorbed decreases slightly with increased branching; in the experiment (see Fig. 3) this was not observed. For concentrations exceeding the $\mathrm{CMC}$, the IFT decreases slightly with increasing degree of branching, as observed experimentally (see Fig. 3). The experiment and the SCFA calculations show that the effect of branching on IFT is subtler than the effect of chain length.

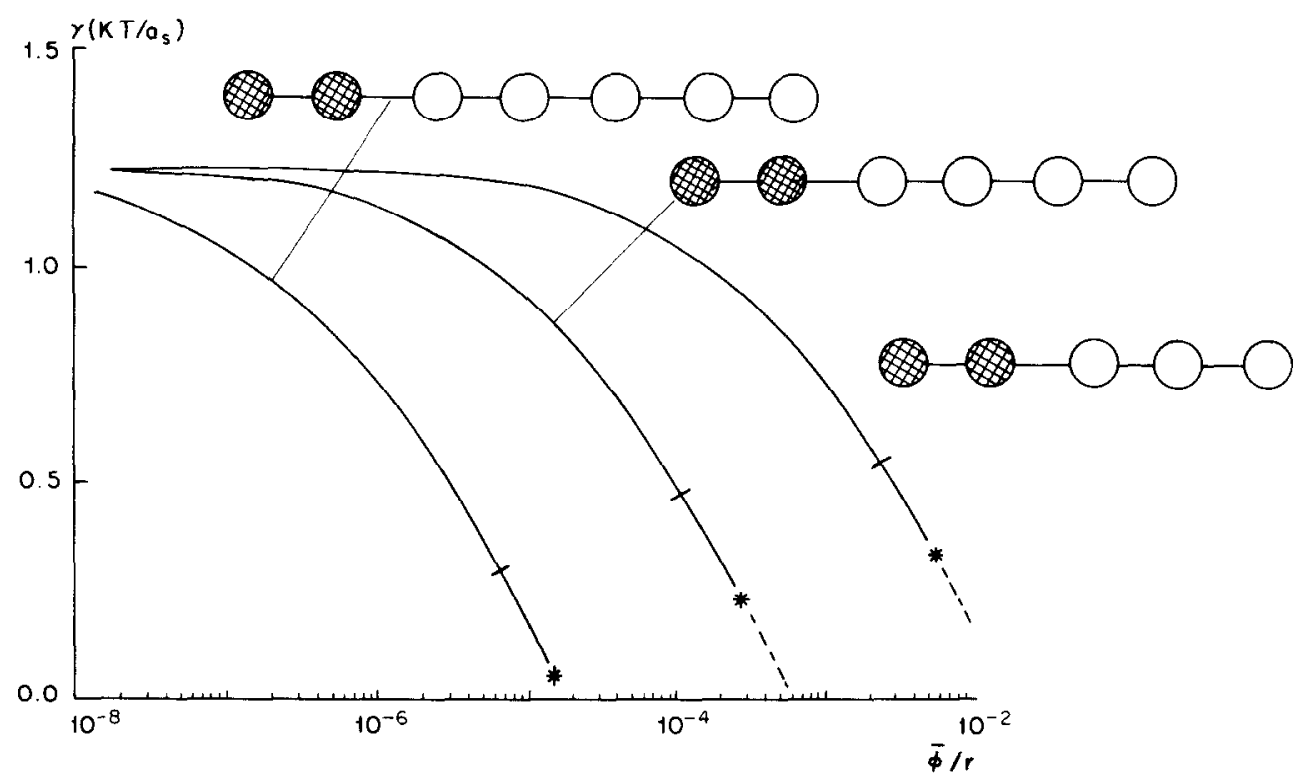

Fig. 4. Interfacial tension as a function of $\bar{\phi} / r$ for $A_{n} B_{2}$ molecules; see text for parameters. 


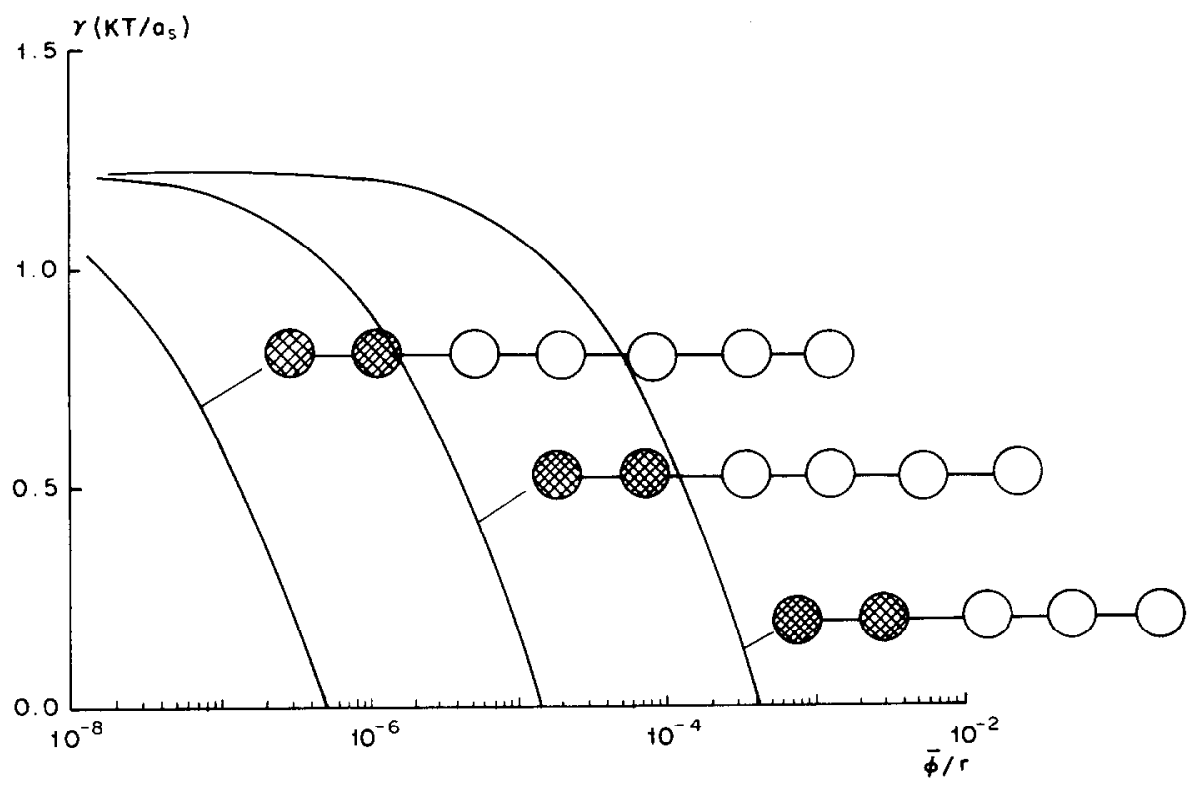

Fig. 5. Interfacial tension as a function of $\phi / r$ for $A_{n} B_{2}$ molecules, $\chi(B W)=0$; see text for other parameters.

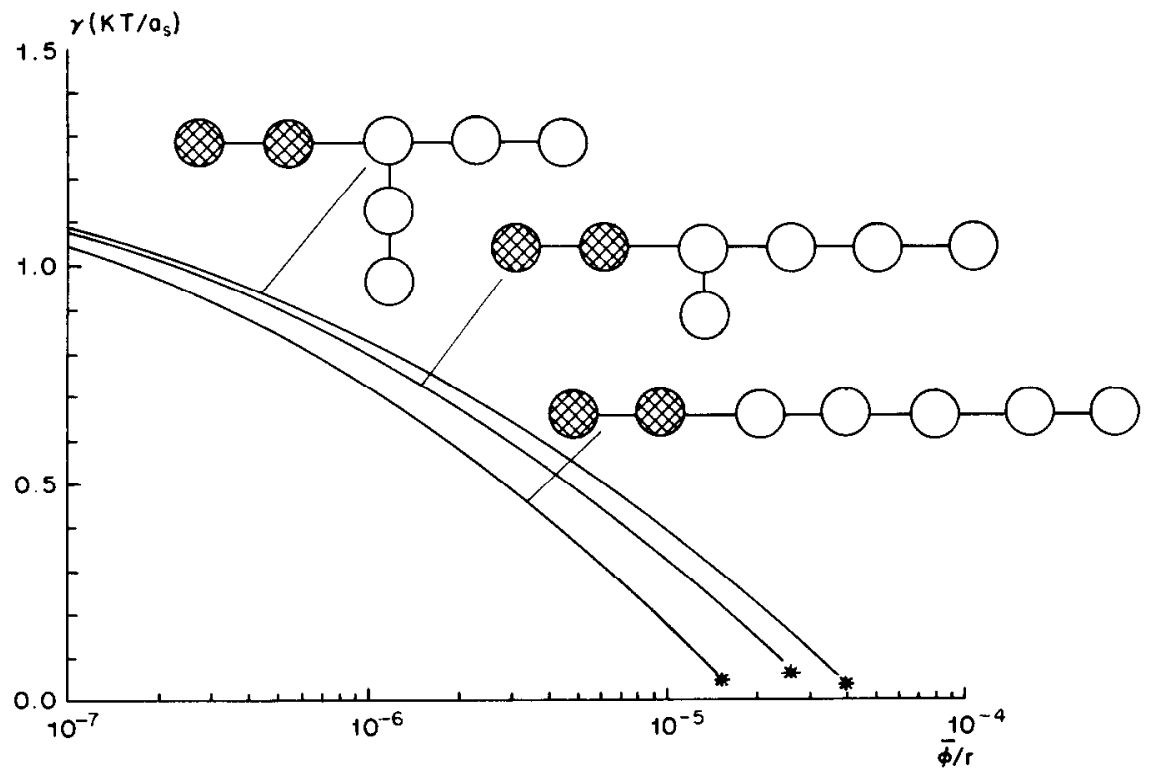

Fig. 6. Interfacial tension as a function of $\phi / r$ for $A_{5} B_{2}$ isomers; same parameters as in Fig. 4.

\subsubsection{Structure of the interfacial region}

Information on the structure of the interfacial region can be obtained from the volume fraction profiles. By way of example, we show volume fraction profiles of $A_{5} B_{2}, W$, and $A_{4}$ at an equilibrium bulk volume fraction of $10^{-4}$, i.e. at the CMC, in Fig. 7(a). The head groups are located at the $\mathrm{W}$-rich side and the tails at the $\mathrm{A}_{4}$-rich side. 

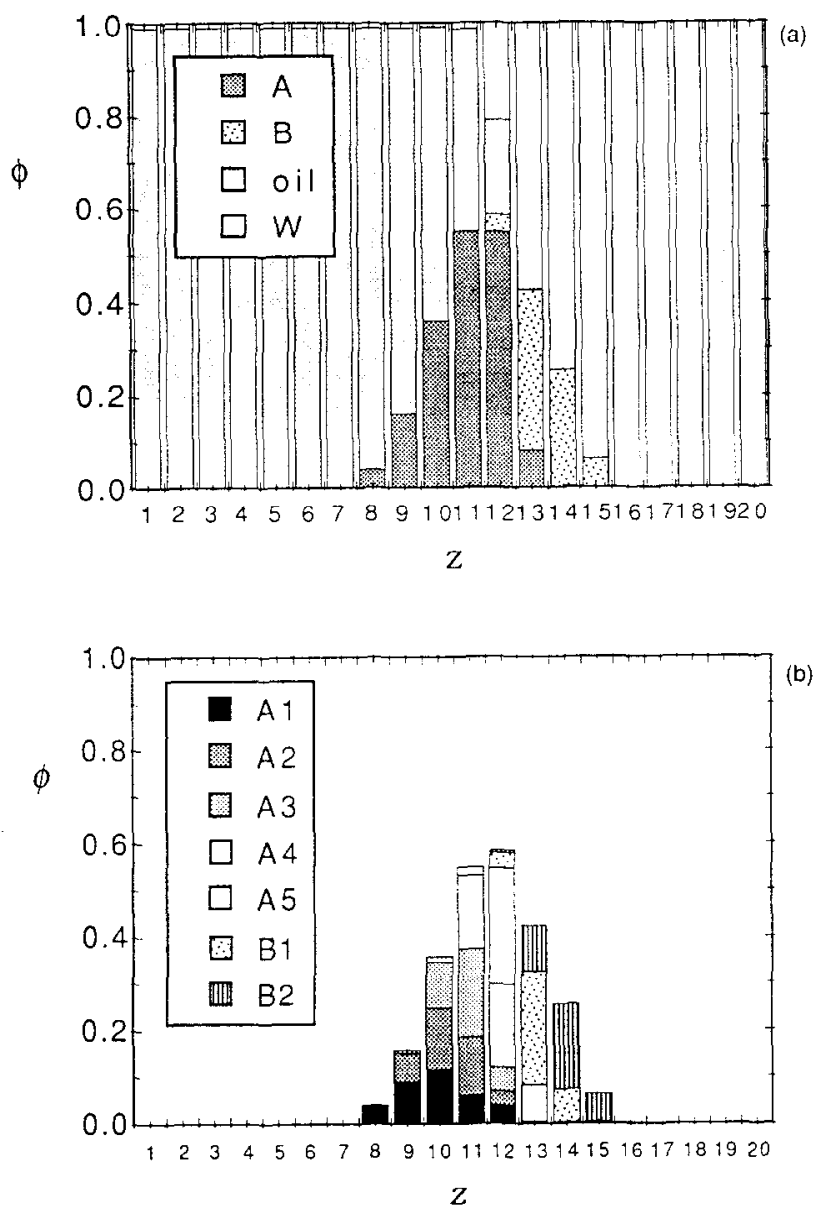

Fig. 7. (a) Volume fraction profiles for W, $A_{4}$ and $A_{5} B_{2}$ head and tail segments at a bulk volume fraction of $A_{5} B_{2}$ of $10^{-4}$. (b) Volume fraction profiles of the individual segments of $A_{5} B_{2}$; same parameters as in Fig. 4.

Considerable head-tail overlap occurs. Figure 7(b) shows the volume fractions of the individual surfactant segments. In particular, the end segment of the tail $\left(A_{1}\right)$ has a rather broad distribution. The distributions of the $A$ segments close to the head group $\left(\mathrm{A}_{4}\right.$ and $\left.\mathrm{A}_{5}\right)$ arc narrowcr, as is the distribution of the head group segments, especially $B_{1}$.

\section{Molecular dynamics (MD) simulations}

\subsection{The model}

Details of the model used in these simulations and the method of calculation have appeared else- where [22-25], so that we shall confine ourselves to a brief description here. The model assumes the existence of two types of particles namely "oil-like" and "water-like". These two types of particles are used to model three types of molecules, namely oil molecules, water molecules and surfactant molecules. An oil molecule consists of a single oil-like particle, a water molecule consists of a single waterlike particle, and a surfactant molecule is made up of a combination of oil-like and water-like particles. In the surfactant molecule, oil and water particles are joined together by harmonic potentials. The two types of particles interact through truncated Lennard-Jones potentials with energy 
parameter $\epsilon_{i j}$, distance parameter $\sigma_{i j}$, and cut-off radius $R_{i j}^{\mathrm{c}}$.

$\Phi_{i j}= \begin{cases}\phi_{i j}(r)-\phi_{i j}\left(R_{i j}^{\mathrm{c}}\right) & r \leqslant R_{i j}^{\mathrm{c}} \\ 0 & r \geqslant R_{i j}^{\mathrm{c}}\end{cases}$

$\phi_{i j}=4 c_{i j}\left[\left(\frac{\sigma_{i j}}{r}\right)^{12}-\left(\frac{\sigma_{i j}}{r}\right)^{6}\right]$

where $i$ and $j$ indicate the type of atom (water or oil) and $r$ is the distance between the atoms. We have assumed that for all interactions $\epsilon_{i j}=\epsilon$ and $\sigma_{i j}=\sigma$. In order to make the $\mathrm{o}-\mathrm{o}$ interactions different from the $\mathrm{w}-\mathrm{o}$ interactions, the potential $\left(R_{i j}^{\mathrm{c}}\right)$ is truncated depending on the type of interaction. The $\mathrm{w}-\mathrm{w}$ and $\mathrm{o}-\mathrm{o}$ interactions are truncated at $R_{i j}^{\mathrm{c}}=2.5 \sigma$ and the o-w interaction at $R_{i j}^{\mathrm{c}}=$ $2^{1 / 6} \sigma$, which makes the latter completely repulsive. As a result, it turns out that at a temperature $T=$ $1.0 \epsilon / k_{\mathrm{B}}$, the oil and water do not mix but form a stable liquid/liquid interface [22,32]. The surfactant molecules are of an amphiphilic nature, with one end being hydrophobic, the other hydrophilic.

With this model it is simple to simulate changes in the structure of the surfactant. For example, addition of oil-like particles to the tail allows us to study the effect of increasing the chain length or of increasing the degree of branching on physicochemical properties.

\subsection{Computational details}

In these simulations we have studied a system consisting of two liquid layers: one contains $256 \mathrm{w}$ particles, the other 512 o particles. These were placed on an f.c.c. lattice at the centre of a box of size $7.15 \sigma \times 7.15 \sigma \times 21.45 \sigma$. Periodic boundary conditions are imposed in all three directions. Surfactants wcre introduced in the following way. First, randomly chosen $w$ and o particles were connected with a harmonic spring. Initially, the spring constant was small, then it was slowly increased to its desired value. After the distance between two neighbouring surfactant particles had reached its equilibrium value, the surfactants were "dragged" to the interfaces, half of the total number of surfactants to each interface. The equations of motion were solved using Verlet's integration scheme [33]. Tests showed that a time step of length $\Delta t=0.005 \tau_{0}$ with $\tau_{0}=\sigma(m / \epsilon)^{1 / 2}$ was appropriate. At least $1.5 \times 10^{6}$ time steps were allowed for equilibration. This was sufficient to obtain stable density profiles and a constant normal component of the pressure tensor (see next paragraph).

We continued a few simulations three times as long, but did not observe any significant deviations in the end results. Of course, this does not guarantee that equilibrium was reached. However, in each simulation a number of surfactants left the interface. This indicates that the simulation time was sufficient for surfactants to diffuse away from the interfacial region.

\subsection{Calculation of the interfacial tension}

In a homogeneous system at equilibrium the thermodynamic pressure is constant and equal in all directions. For an inhomogeneous system, hydrodynamic equilibrium requires that the component of the pressure tensor normal to the interface be constant throughout the system. The components tangential to the interface can vary in the interfacial region, but must be equal to the normal component in the bulk liquids.

For an inhomogeneous system there is no unambiguous way of calculating the normal and tangential components of the pressure tensor. Here we have used the Kirkwood-Buff convention [34].

The interfacial tension can be calculated by integrating the difference of the normal and tangential components of the pressure tensor across the interface; for details see Refs. 23 and 25.

\subsection{Results}

Figure 8 is a plot of the interfacial tension as a function of the total number of surfactants; numerical values are given in Table 2 . It is not possible to determine an accurate surfactant concentration in these simulations, hence we have resorted to a linear concentration scale along the horizontal axis 


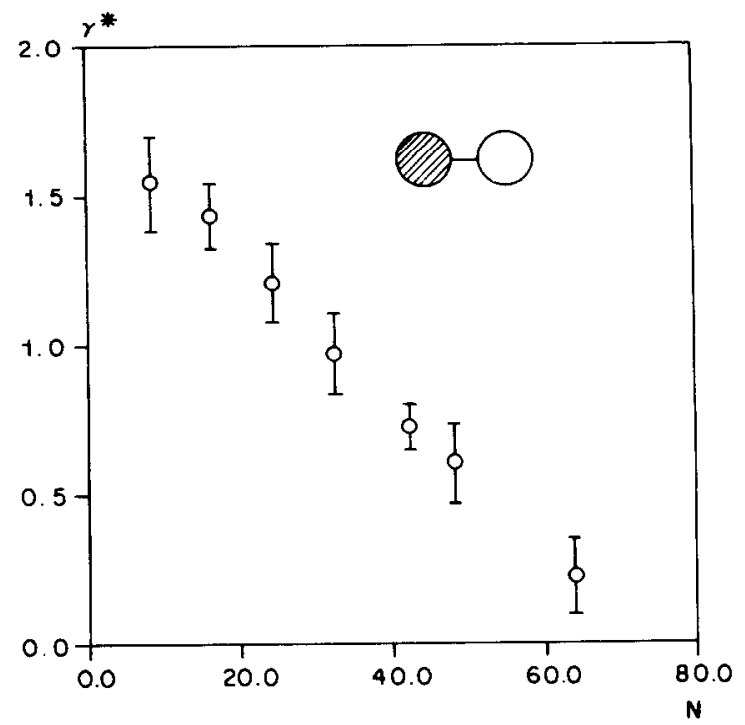

Fig. 8. Reduced interfacial tension $\gamma^{*}=\gamma \sigma^{2} \epsilon^{-1}$ as a function of the total number of surfactants $(N)$ for a dumb-bell molecule, as obtained from the molecular dynamics simulations.

Table 2

The interfacial tension $\gamma$ for linear and branched surfactants from MD simulations ( $N$ is the total number of surfactants)

\begin{tabular}{|c|c|c|c|c|c|}
\hline \multirow[t]{3}{*}{$N$} & \multicolumn{5}{|c|}{ Interfacial tension $\gamma$} \\
\hline & \multicolumn{3}{|c|}{ Linear $^{\mathbf{a}}$} & \multicolumn{2}{|c|}{ Branched $^{a}$} \\
\hline & ow & $\mathrm{O}_{3} \mathrm{w}$ & $\mathrm{O}_{5} \mathrm{~W}$ & $\mathrm{O}_{2}\left(\mathrm{O}_{1}\right) \mathrm{W}$ & $\mathrm{O}_{3}\left(\mathrm{o}_{2}\right) \mathrm{w}$ \\
\hline 0 & 1.68 & 1.68 & 1.68 & 1.68 & 1.68 \\
\hline 8 & 1.55 & & & & \\
\hline 12 & 1.50 & 1.29 & 1.20 & 1.28 & 1.30 \\
\hline 16 & 1.44 & & & & \\
\hline 24 & 1.21 & & & & \\
\hline 32 & 0.96 & 0.75 & 0.66 & 0.68 & 0.97 \\
\hline 42 & 0.73 & 0.56 & 0.36 & 0.54 & 0.70 \\
\hline 48 & 0.61 & & & & \\
\hline 64 & 0.23 & & & & \\
\hline
\end{tabular}

${ }^{a}$ Surfactant structures (except $o_{2}\left(o_{1}\right) w$ ) shown in Figs. 8-10.

in Fig. 8. It can be seen that the reduction of the IFT is proportional to the number of surfactant particles. Because of our relatively small system, we could not extend these simulations to a larger number of surfactants in order to verify the formation of micelles. However, the model is capable of predicting micelle formation in systems comprising larger numbers of particles, as has been described elsewhere in detail ([24,25] and the Discussion section of this paper). Figure 9 shows that the model predicts that increasing the tail length leads to a decrease of interfacial tension for the same total number of surfactant particles in the system, in agreement with the experimental data in Fig. 2 and with the SCFA calculations in Fig. 4. Numerical values are given in Table 2.

The effect of chain branching on interfacial tension is shown in Table 2 and in Fig. 10. The simulation shows that branched surfactants are less efficient than linear compounds with the same number of carbon atoms, in agreement with the experimental data in Fig. 3 and with the SCFA calculations in Fig. 6. According to the simulation, a branched surfactant with five tail segments has approximately the same effect on interfacial tension as one with only one tail segment.

It is interesting that for three tail segments no difference was observed between a branched and a linear surfactant. This suggests that the low surface activity of the branched surfactant with five tail segments may be caused primarily by its enhanced solubility in the oil phase.

\section{Discussion}

For the comparison between SCFA calculations and MD simulations we must bear in mind that apart from differences in theory, there are also differences in the choice of parameters. An important difference is that in SCFA the head group segments are preferentially solvated $(\chi(\mathrm{BW})=$ $-4.3)$ which creates a repulsion between the head groups and stimulates micelle formation. In the SCFA calculations with $\chi(\mathrm{BW})=0$ this effective repulsion is absent and no regular miccllcs are formed. In the MD simulations, where no head group repulsion was taken into account, micelle formation was not observed in the small system discussed in this paper. Later MD simulations $[24,25]$ on a network of 100 transputers using a parallel MD algorithm showed that in much larger systems (39000 particles) the spontaneous forma- 


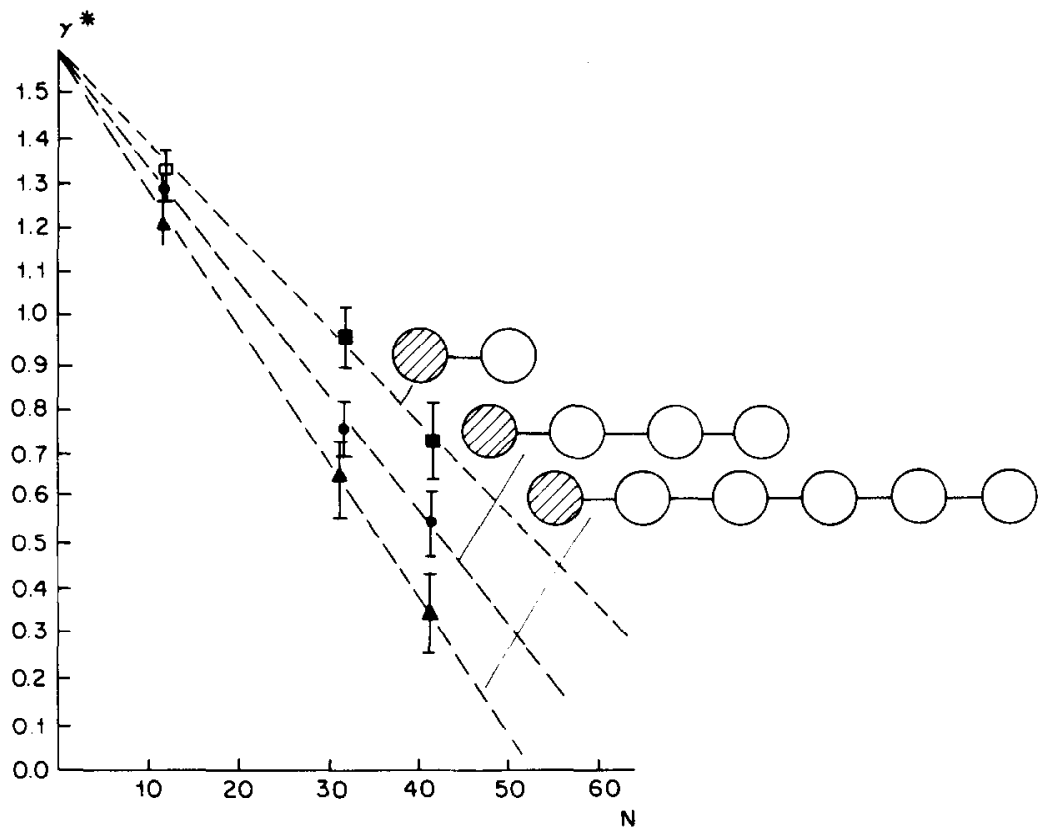

Fig. 9. Reduced interfacial tension $\gamma^{*}=\gamma \sigma^{2} \epsilon^{-1}$ as a function of the total number of surfactants $(N)$ for various chain lengths as obtained from the molecular dynamics simulations.

tion of micelles can indeed be observed. Recent MD simulations [35] have shown that the complete micelle size distribution can also be obtained, as well as dynamic processes such as the entering of single surfactants into micelles, single surfactants leaving micelles, the fusion of two micelles, and the slow breakdown of micelles.

Density profiles across the interfacial region calculated by means of the SCFA theory and by MD simulations [24,25] lead to the observations that the end segments of the surfactant tails have a rather broad distribution and that considerable head-tail overlap can occur.

In the SCFA calculations it is assumed that the volume is totally filled with either $\mathrm{A}_{n} \mathrm{~B}_{2}, \mathrm{~W}$ or $\mathrm{A}_{4}$. From the MD simulations [25] it appears that this is an oversimplification. Variations in total density can be taken into account in the SCFA theory by introducing an additional monomeric component called "vacancies", V. The sum of the volume fractions is still unity, because the vacancies too, have a volume fraction in each layer. As $\mathrm{V}$ is assumed to have a density of zero, variations in density are now allowed. The $\chi$ parameters between $\mathrm{V}$ and the other components can be estimated by modelling the liquid/vapour equilibria of, say, decane and water. This yields $\chi$ values of $\chi(\mathrm{BV})=$ 3.1 and $\chi(\mathrm{AV})=3.5$. The volume fraction profiles of $\mathrm{A}, \mathrm{B}, \mathrm{W}$ and $\mathrm{A}_{4}$ are shown in Fig. 11. In agreement with the $\mathrm{MD}$ simulations [24,25] we observe a local minimum in the density distribution at the liquid/liquid interface. Similar density distribution functions have been observed by means of neutron reflection measurements at water/air and water/oil interfaces in the presence of surfactants $[36,37]$. A new aspect of the work presented here is that SCFA calculations and MD simulations describe the detailed structure of the interface, as well as micellar structurcs, whercas prcvious theoretical studies have been concerned with either isolated micelles or interfacial layers.

An important advantage of the SCFA theory over the MD simulations is that the calculation times are much shorter. Typically, 5 min CPU on a VAX 8700 machine were required to calculate a volume fraction profile. Even if a series of calcula- 


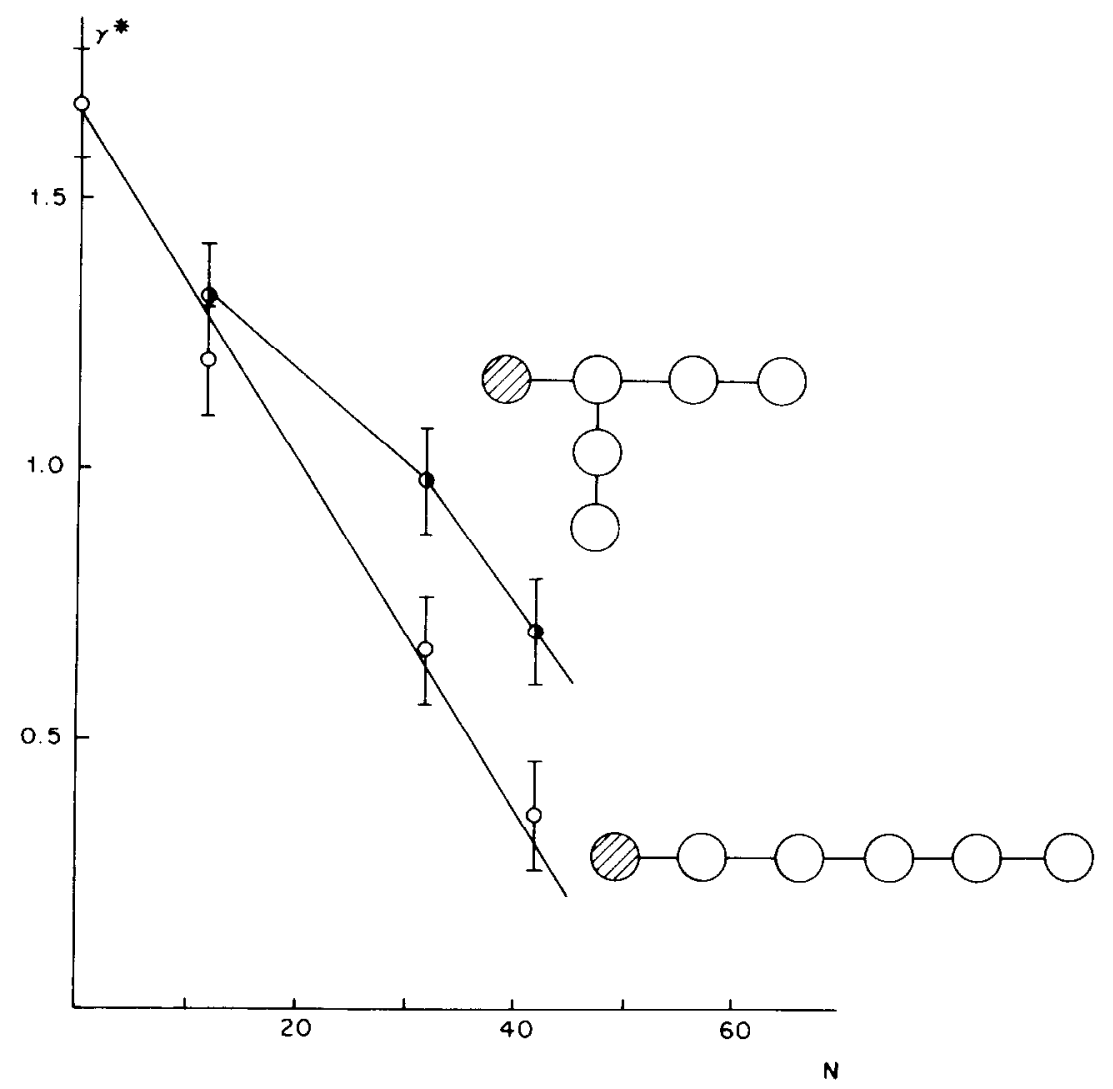

Fig. 10. Reduced interfacial tension $\gamma^{*}=\gamma \sigma^{2} \epsilon^{-1}$ as a function of the total number of surfactants $(N)$ for a linear and a branched molecule as obtained from the molecular dynamics simulations.

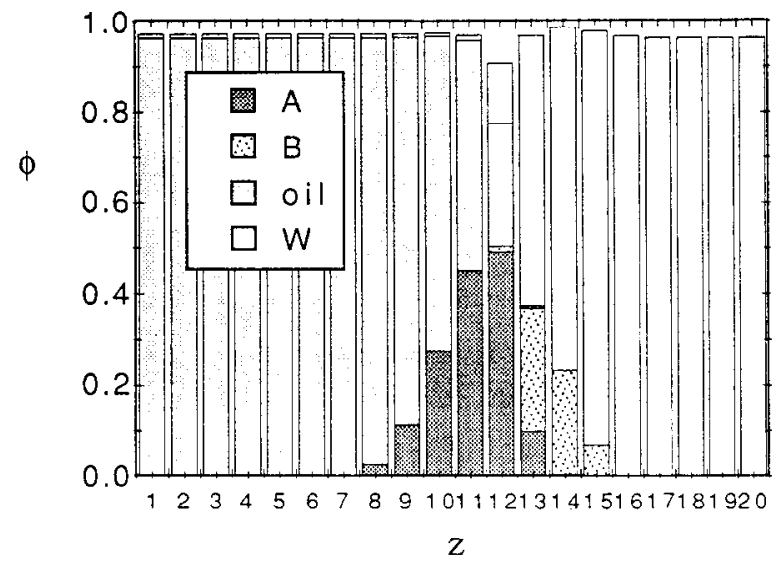

Fig. 11. Volume fraction profiles for $W, \mathbf{A}_{4}$ and $\mathbf{A}_{5} \mathbf{B}_{2}$ head and tail segments in the presence of component $V$, representing free volume; same parameters as in Fig. $4(\chi(\mathrm{WV})=3.1$ and $\chi(\mathrm{AV})=3.5)$. tions had to be carried out to find the "artefactfree" solution, the compulation time was short compared with that required for the MD simulations. As in SCFA theory calculations take little time, it is easier to arrive at a choice of parameters that represents surfactant behaviour as observed in the experiments. Another advantage is that equilibrium is guaranteed. The main disadvantage of the SCFA theory is that a lattice is used and a mean field approximation is made in each lattice layer parallel to the interface.

The advantages of the MD simulation are that no lattice is required and inhomogeneities in more than one dimension can be investigated. Furthermore, dynamic processes such as the kinetics of micelle formation lend themselves easily to the technique of MD simulation. An important aspect is that MD simulations describe the detailed 
structure of the interface as well as micellar structures, whereas previous theoretical studies have been concerned with either isolated micelles or interfacial layers. The main disadvantage is that it is not easy to check the existence of an equilibrium situation. Comparison of the two methods is useful to establish the influence of the lattice and the mean field approach on the one hand, and to establish whether equilibrium has been reached on the other.

\section{Conclusions}

Experimental measurements, molecular dynamics simulations, and self-consistent field calculations of surfactant adsorption at an oil/water interface all show that (1) increasing the chain length of a surfactant lowers the IFT at a given surfactant concentration; (2) increasing chain branching of a surfactant increases the IFT at a given surfactant concentration.

For model surfactants, the relation between the structure of a surfactant and its interfacial tension at an oil/water interface can therefore be predicted in a qualitative sense. The formation of micelles can also be predicted with self-consistent field calculations and MD simulations.

It is encouraging that theoretical models provide such reasonable descriptions of complex colloidal systems. The authors hope that this will lead to increased collaboration between physicists and colloid scientists.

\section{References}

1 T. Young, in G. Peacock (Ed.), Miscellaneous Works, Vol. 1, Murray, London, 1855, p. 418.

2 J.W. Gibbs, The Collected Works of J.W. Gibbs, Vol. I, Longmans-Green, New York, 1931, p. 219.

3 E.I. Franses, J.E. Puig, Y. Talmon, W.G. Miller, L.E. Scriven and H.T. Davis, J. Phys. Chem., 84 (1980) 1547.

4 J.M.H.M. Scheutjens and G.J. Fleer, J. Phys. Chem., 83 (1979) 1619.

5 J.M.H.M. Scheutjens and G.J. Fleer, J. Phys. Chem., 84 (1980) 178.

6 O.A. Evers, J.M.H.M. Scheutjens and G.J. Fleer, Macromolecules, 23 (1990) 5221.
7 F.A.M. Leermakers and J.M.H.M. Scheutjens, J. Chem. Phys., 5 (1988) 3264.

8 F.A.M. Leermakers and J.M.H.M. Scheutjens, J. Colloid Interface Sci., 136 (1990) 231.

9 F.A.M. Leermakers and J.M.H.M. Scheutjens, J. Phys. Chem., 93 (1989) 7417.

10 M.R. Böhmer, L.K. Koopal and J. Lyklema, J. Phys. Chem., 95 (1991) 9569.

11 J.M.H.M. Scheutjens, F.A.M. Leermakers, N.A.M. Besseling and J, Lyklema, in K.L. Mittal (Ed.), Surfactants in Solution, Vol. 7, Plenum, New York, 1989.

12 M.R. Böhmer and L.K. Koopal, Langmuir, 6 (1990) 1478.

13 M.R. Böhmer, L.K. Koopal, R. Janssen, E.M. Lee, R.K. Thomas and A.R. Rennie, Langmuir, 8 (1992) 2228.

14 M.R. Böhmer and L.K. Koopal, Langmuir, 8 (1992) 2649.

15 M.R. Böhmer and L.K. Koopal, Langmuir, 8 (1992) 2660.

16 D.W.R. Gruen, J. Phys. Chem., 89 (1985) 146.

17 1. Szleifer, Ph.D. Thesis, Hebrew University, Jerusalem, 1988.

18 I. Szleifer, A. Ben-Shaul and W.M. Gelbart, J. Chem. Phys., 86 (1987) 7094.

19 P. Van der Ploeg and H.J.C. Berendsen, Mol. Phys., 49 (1983) 233.

20 P.J. Lindse, J. Chem. Phys., 86 (1987) 4177.

21 I.L. Carpenter and W.J. Hehre, J. Phys. Chem., 94 (1990) 531.

22 B. Smit, Phys. Rev. A, 37 (1988) 3431.

23 B. Smit, A.G. Schlijper, L.A.M. Rupert and N.M. Van Os, J. Phys. Chem., 94 (1990) 6934.

24 B. Smit, P.A.J. Hilbers, K. Esselink, L.A.M. Rupert, N.M. Van Os and A.G. Schlijper, Nature, 348 (1990) 624.

25 B. Smit, P.A.J. Hilbers, K. Esselink, L.A.M. Rupert, N.M. Van Os and A.G. Schlijper, J. Phys. Chem., 95 (1991) 6361 .

26 N.M. Van Os, G.J. Daane and T.A.B.M. Bolsman, J. Colloid Interface Sci., 115 (1987) 402.

27 N.M. Van Os, G.J. Daane and T.A.B.M. Bolsman, J. Colloid Interface Sci., 123 (1988) 267.

28 N.M. Van Os, G.J. Daane and G. Haandrikman, J. Colloid Interface Sci., 141 (1991) 199.

29 W. Binana-Limbele, N.M. Van Os, L.A.M. Rupert and R. Zana, J. Colloid Interface Sci., 141 (1991) 157.

30 P. Mukerjee and K. Mysels, J. Natl. Stand. Ref. Data Ser., U.S. Natl., Bur. Stand., No. 36, 1971.

31 P.J. Flory, Principles of Polymer Chemistry, Cornell University Press, Ithaca, NY, 1953.

32 M.M. Telo da Gama and K.E. Gubbins, Mol. Phys., 59 (1986) 227.

33 L. Verlet, Phys. Rev,, 159 (1967) 98.

34 M.J.P. Nijmeijer, Ph.D. Thesis, Rijksuniversiteit Leiden, 1990.

35 B. Smit, K. Esselink, P.A.J. Hilbers, N.M. Van Os, L.A.M. Rupert and I. Szleifer, Langmuir, 9 (1993) 9.

36 E.A. Simister, E.M. Lee, R.K. Thomas and J. Penfold, J. Phys. Chem., 96 (1992) 1373.

37 T. Cosgrove, J.S. Phipps and R.M. Richardson, Colloids Surfaces, 62 (1992) 199. 